\title{
Molecular fingerprinting reflects different histotypes and brain region in low grade gliomas
}

\author{
Samantha Mascelli ${ }^{{ }^{*}+}$, Annalisa Barla ${ }^{2 \dagger}$, Alessandro Raso ${ }^{1}$, Sofia Mosci ${ }^{2}$, Paolo Nozza ${ }^{3}$, Roberto Biassoni ${ }^{4}$, \\ Giovanni Morana ${ }^{5}$, Martin Huber ${ }^{6}$, Cristian Mircean ${ }^{6}$, Daniel Fasulo ${ }^{7}$, Karin Noy ${ }^{7}$, Gayle Wittemberg ${ }^{7}$, Sara Pignatelli ${ }^{8}$, \\ Gianluca Piatelli', Armando Cama ${ }^{1}$, Maria Luisa Garré ${ }^{8}$, Valeria Capra ${ }^{1}$ and Alessandro Verri ${ }^{2}$
}

\begin{abstract}
Background: Paediatric low-grade gliomas (LGGs) encompass a heterogeneous set of tumours of different histologies, site of lesion, age and gender distribution, growth potential, morphological features, tendency to progression and clinical course. Among LGGs, Pilocytic astrocytomas (PAs) are the most common central nervous system (CNS) tumours in children. They are typically well-circumscribed, classified as grade I by the World Health Organization (WHO), but recurrence or progressive disease occurs in about 10-20\% of cases. Despite radiological and neuropathological features deemed as classic are acknowledged, PA may present a bewildering variety of microscopic features. Indeed, tumours containing both neoplastic ganglion and astrocytic cells occur at a lower frequency.
\end{abstract}

Methods: Gene expression profiling on 40 primary LGGs including PAs and mixed glial-neuronal tumours comprising gangliogliomas (GG) and desmoplastic infantile gangliogliomas (DIG) using Affymetrix array platform was performed. A biologically validated machine learning workflow for the identification of microarray-based gene signatures was devised. The method is based on a sparsity inducing regularization algorithm $l_{1} l_{2}$ that selects relevant variables and takes into account their correlation. The most significant genetic signatures emerging from gene-chip analysis were confirmed and validated by qPCR.

Results: We identified an expression signature composed by a biologically validated list of 15 genes, able to distinguish infratentorial from supratentorial LGGs. In addition, a specific molecular fingerprinting distinguishes the supratentorial PAs from those originating in the posterior fossa. Lastly, within supratentorial tumours, we also identified a gene expression pattern composed by neurogenesis, cell motility and cell growth genes which dichotomize mixed glial-neuronal tumours versus PAs. Our results reinforce previous observations about aberrant activation of the mitogen-activated protein kinase (MAPK) pathway in LGGs, but still point to an active involvement of TGF-beta signaling pathway in the PA development and pick out some hitherto unreported genes worthy of further investigation for the mixed glial-neuronal tumours.

Conclusions: The identification of a brain region-specific gene signature suggests that LGGs, with similar pathological features but located at different sites, may be distinguishable on the basis of cancer genetics. Molecular fingerprinting seems to be able to better sub-classify such morphologically heterogeneous tumours and it is remarkable that mixed glial-neuronal tumours are strikingly separated from PAs.

Keywords: Gene expression profile, Machine learning, Low-grade glioma, PA, Mixed glial-neuronal tumours

\footnotetext{
* Correspondence: SamanthaMascelli@ospedale-gaslini.ge.it

†Equal contributors

${ }^{1}$ Neurosurgery Unit, Istituto Giannina Gaslini, via G. Gaslini 5, 16147, Genoa, Italy

Full list of author information is available at the end of the article
}

\section{Ciomed Central}

(c) 2013 Mascelli et al.; licensee BioMed Central Ltd. This is an Open Access article distributed under the terms of the Creative Commons Attribution License (http://creativecommons.org/licenses/by/2.0), which permits unrestricted use, distribution, and reproduction in any medium, provided the original work is properly cited. 


\section{Background}

Primary intra-axial paediatric low grade tumours include pilocytic astrocytoma (PA), pilomyxoid astrocytoma, diffuse fibrillary astrocytoma (FA), ganglioglioma (GG), desmoplastic infantile ganglioglioma (DIG), desmoplastic infantile astrocytoma (DIA) and dysembryoplastic neuroepithelial tumour [1]. For brevity, they will be thereafter defined LGG.

PA is the most common central nervous system (CNS) tumour, representing approximately 21-23\% of all primary brain tumours in children [2]. It is typically a well-circumscribed, contrast-enhancing astrocytic neoplasm with prolonged overall survival and high complete remission rates [3]. PA arises most commonly in the cerebellum, but can be found anywhere, including the cerebral hemispheres, thalamus and hypothalamus, brainstem, optic pathways, and spinal cord $[3,4]$.

Reflecting the generally slow growth and low proliferative potential of LGGs, complete surgical resection is the preferred therapeutic choice. Unfortunately, gross total resection is not attainable in many of these tumours that are centrally located which, in about 10 to $20 \%$ of the cases, despite adjuvant treatment, tend to recur or show progressive growth [5,6]. Most importantly, rare examples of PA undergo malignant transformation, even if completely resected $[7,8]$.

Despite radiological and neuropathological features deemed as classic are acknowledged, PA may present a bewildering variety of microscopic features, including a wide range of tissue patterns, most of which may be found within the same lesion. It is worth remembering that both normal and neoplastic astrocytes exhibit molecular and functional heterogeneity [9-13].

The tumours containing both neoplastic ganglion and astrocytic cells are rare, representing less than an hundredth of the tumours of CNS and its coverings. Such tumours, which belong to the neuronal and mixed glial-neuronal tumours of the WHO classification and corresponding to grade I, comprise gangliogliomas (GG), and gangliogliomas with desmoplasia, i.e. desmoplastic infantile gangliogliomas (DIG) both typically arising from the telencephalon [2]. The differential diagnosis may be difficult due to small biopsy size. Moreover, the glial component of a ganglioglioma may be pilocytic looking (this is as much as to say that a pilocytic morphology is considered completely acceptable in a ganglioglioma). Lack of specific immunohistochemical, cytogenetic, or molecular markers increases difficulties in classification.

The expanding utilization of high-throughput technologies to study paediatric brain tumours will likely change how they are both classified and treated henceforward $[5,6]$. In this field, the use of microarrays has been expanding exponentially to several areas such as genetic screening, safety assessment and diagnostics [14], but repeatability of published microarray studies is apparently limited $[15,16]$. In the neuro-oncological context, a LGG genotype-phenotype correlation still remains an open problem $[17,18]$. Gene signatures able to classify LGGs in accordance with clinical and biological features were provided [9,19-22]. Nevertheless, a complete genetic landscape of paediatric PA is still missing and the specific molecular signatures able to correlate their phenotype (brain sites and heterogeneous histotypes) to their genotype still remain to be studied in depth.

Keeping this in mind, we aimed to identify a molecular fingerprinting able to reflect different histotypes and brain region in LGGs. In particular, the study addressed three different biological questions: (1) characterize supratentorial vs. infratentorial LGGs, (2) identify a specific characterization for the PAs based upon site of lesion, and (3) discriminate, within supratentorial neoplasms, mixed glial-neuronal tumours vs. PAs.

This relatively simple, albeit fraught with meaning, goal gave us the opportunity to develop a robust and validated experimental workflow, paving the way for future studies, whose goal will be the identification of gene fingerprints explicitly correlated to clinical parameters.

\section{Methods}

We adopted a biologically validated method to identify reliable and predictive gene expression signatures on tumour data. The pipeline, represented in Figure 1, is a supervised machine learning workflow consisting in 3 main consecutive phases: case selection and tumour specimen processing, unbiased $l_{1} l_{2}$ feature selection framework with functional characterization of the gene signature, and real-time quantitative reverse transcription-PCR (qPCR). Detailed description of the pipeline is reported in Additional file 1.

\section{Case selection and tumour processing}

A series (dataset 1) of 40 paediatric primary LGGs who underwent surgery from 1991 to 2009 at the Neurosurgery Unit of the Giannina Gaslini Children's Hospital were selected and enrolled in the study. The inclusion criteria were diagnosis of PA or ganglioglioma with or without desmoplasia, i.e. GG or DIG; the availability of complete clinical data and fresh frozen tissue specimen with a tumour cell content of at least $80 \%$, while exclusion criteria were lack of histological diagnosis and the presence of extensive dissemination. The cohort included 27 PAs, 12 mixed glial-neuronal tumours (7 GGs and 5 DIGs) and one FA. Seventeen tumours arose in infratentorial regions, while 23 were supratentorial. The FA was forced in the first phase of analysis and partially during the second phase in order to have an internal control. Only one case was associated with a genetic syndrome, namely Neurofibromatosis type 1 (NF1) [MIM ID \#162200]. The male/female 


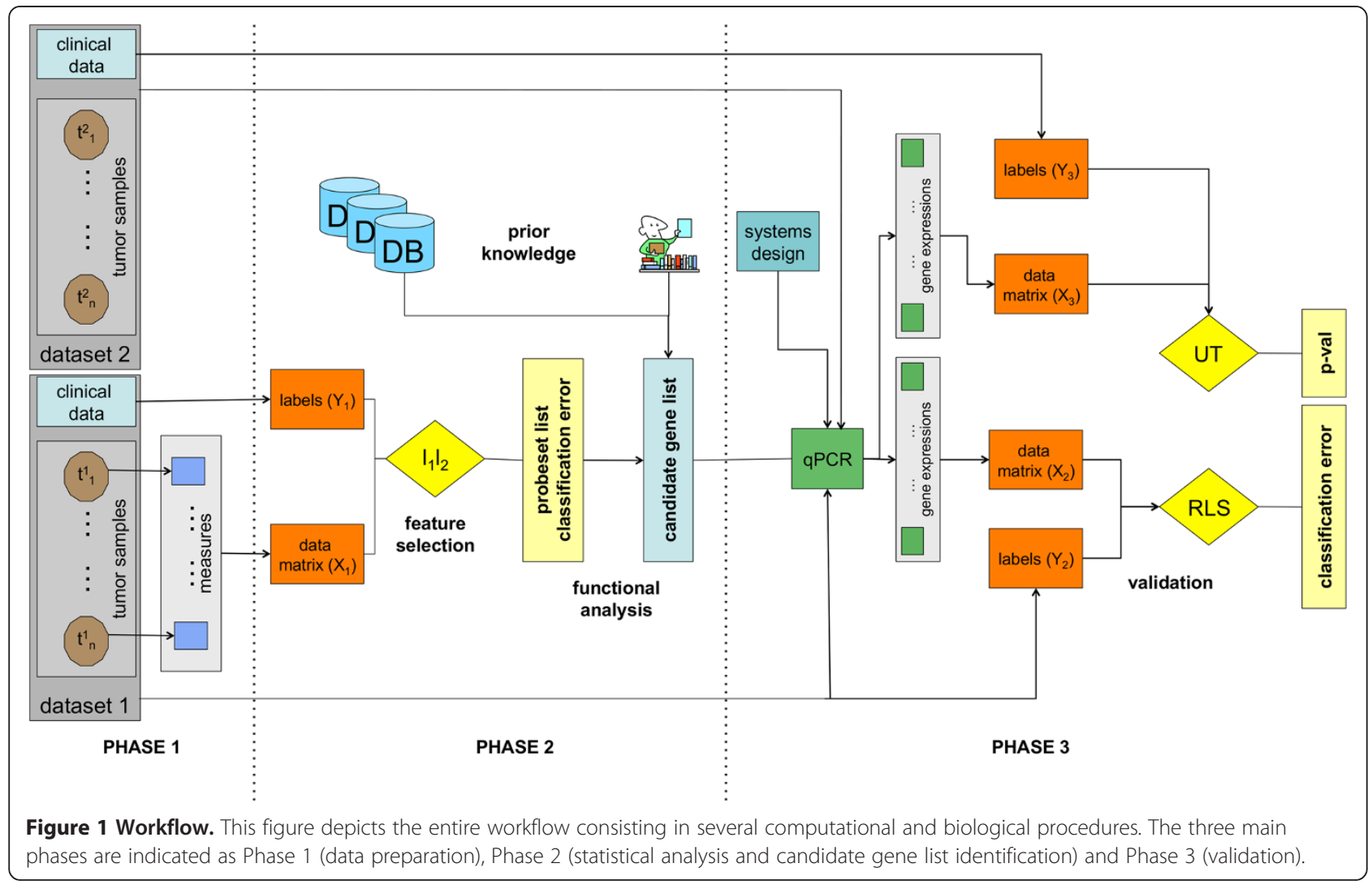

ratio of $1.2: 1$, and the mean age 7 years (range 5 months to 17 years). The main clinical-pathological features are summarized in Table 1. The sections were reviewed by the local neuropathologist (P.N.) and the tumours were classified according to the WHO classification [3]. The sets of samples are formed to precisely answer the biological questions of interest. Moreover, the sets were made the more homogeneous possible in order to minimize the undesiderable effects of the inter-tumoural genetic differences due to the intrinsic constitutional variations among individuals.

Total RNA was extracted from serial frozen sections of tumour tissue by using the TRIzol reagent combined with silica column purification system (Invitrogen, Carlsbad, CA). Quantification and quality assurance were performed using the NanoDrop spectrophotometer (NanoDrop Technologies Wilmington, Delawere USA) and the Agilent 2100 bioanalyzer (Agilent Technologies, Waldbronn, Germany), respectively. Double-stranded cDNA were processed according to the Affymetrix GeneChip Expression Analysis Technical Manual (Affymetrix, Santa Clara, CA). Microarray data for 40 LGG samples was generated with Affymetrix HG-U133Plus2.0 arrays (Affymetrix, Santa Clara, CA). Gene expressions were extracted from the .CEL files and normalized using the Robust Multichip Average method [23] by running an $\mathrm{R}$ [24] script, based on the aroma package [25]. The dataset for the microarray experiment was uploaded in the Gene Expression Omnibus public repository at National Center for Biotechnology Information [accession number GSE28238].

Written informed consent was obtained from all the patients'parents or guardians and the local Ethics Committee for human studies approved the research.

\section{Unbiased $I_{1} I_{2}$ feature selection framework}

The feature selection method we adopted is a regularization method capable of selecting subsets of discriminative genes, namely $l_{1} l_{2}$ regularization with double optimization. The algorithm can be tuned to give a minimal set of discriminative genes or larger sets including correlated genes. The method is based on the optimization principle presented in [26] and further developed and studied in $[27,28]$.

The $l_{1} l_{2}$ with double optimization algorithm looks for a linear function (model), whose sign gives the classification rule that can be used to associate a new sample to one of the two classes. The output function is a sparse model, i.e. some input variables (probe-sets) will not contribute to the final estimator. The algorithm is based on the minimization of a functional depending on a least square error term combined with two penalties. The least square 
Table 1 Clinical data

\begin{tabular}{|c|c|c|c|c|c|}
\hline ID & $\begin{array}{c}\text { Age } \\
\text { (months) }\end{array}$ & Gender & Diagnosis & $\begin{array}{l}\text { WHO } \\
\text { grade }\end{array}$ & Site \\
\hline 1 & 35 & $\mathrm{~F}$ & PA & 1 & Infratentorial \\
\hline 2 & 30 & M & PA & I & Infratentorial \\
\hline 3 & 29 & M & PA & 1 & Infratentorial \\
\hline 4 & 38 & $\mathrm{~F}$ & PA & I & Infratentorial \\
\hline 5 & 37 & M & PA & I & Infratentorial \\
\hline 6 & 42 & $\mathrm{~F}$ & PA & 1 & Infratentorial \\
\hline 7 & 16 & $\mathrm{~F}$ & PA & I & Infratentorial \\
\hline 8 & 46 & $\mathrm{~F}$ & PA & I & Infratentorial \\
\hline 9 & 41 & M & PA & I & Infratentorial \\
\hline 10 & 33 & M & PA & I & Infratentorial \\
\hline 11 & 143 & $\mathrm{~F}$ & PA & 1 & Infratentorial \\
\hline 12 & 82 & $\mathrm{~F}$ & PA & 1 & Infratentorial \\
\hline 13 & 165 & $\mathrm{~F}$ & PA & I & Infratentorial \\
\hline 14 & 48 & $\mathrm{~F}$ & PA & I & Infratentorial \\
\hline 15 & 67 & M & PA & I & Infratentorial \\
\hline 16 & 149 & M & PA & 1 & Infratentorial \\
\hline 17 & 36 & $\mathrm{~F}$ & PA & 1 & Infratentorial \\
\hline 18 & 161 & M & PA & 1 & Supratentorial \\
\hline 19 & 104 & $\mathrm{~F}$ & PA & I & Supratentorial \\
\hline 20 & 176 & M & PA & I & Supratentorial \\
\hline 21 & 153 & M & PA & I & Supratentorial \\
\hline 22 & 108 & $\mathrm{~F}$ & PA & I & Supratentorial \\
\hline 23 & 188 & M & PA & 1 & Supratentorial \\
\hline 24 & 128 & $M$ & PA & 1 & Supratentorial \\
\hline 25 & 32 & $\mathrm{~F}$ & PA & I & Supratentorial \\
\hline 26 & 5 & $\mathrm{~F}$ & PA & 1 & Supratentorial \\
\hline 27 & 108 & M & PA & I & Supratentorial \\
\hline 28 & 171 & M & FA & $\|$ & Supratentorial \\
\hline 29 & 11 & $\mathrm{~F}$ & DIG & I & Supratentorial \\
\hline 30 & 27 & $\mathrm{~F}$ & DIG & I & Supratentorial \\
\hline 31 & 6 & $\mathrm{~F}$ & DIG & I & Supratentorial \\
\hline 32 & 6 & M & DIG & I & Supratentorial \\
\hline 33 & 13 & M & DIG & 1 & Supratentorial \\
\hline 34 & 14 & $\mathrm{~F}$ & GG & I & Supratentorial \\
\hline 35 & 155 & M & GG & I & Supratentorial \\
\hline 36 & 119 & $\mathrm{~F}$ & GG & I & Supratentorial \\
\hline 37 & 189 & $\mathrm{~F}$ & GG & I & Supratentorial \\
\hline 38 & 149 & M & GG & I & Supratentorial \\
\hline 39 & 199 & $\mathrm{~F}$ & GG & I & Supratentorial \\
\hline 40 & 130 & $\mathrm{~F}$ & GG & । & Supratentorial \\
\hline
\end{tabular}

For each patient we report the corresponding ID, age at surgery (in months), gender ( $F=$ female, $M=$ male), diagnosis ( $P A=$ pilocytic astrocytoma,

$\mathrm{FA}=$ diffuse fibrillary astrocytoma, DIG = desmoplastic infantile ganglioglioma,

GG = ganglioglioma and site of lesion. Patient ID24 reported in bold is

associated to the genetic syndrome NF1. term ensures fitting of the data whereas adding the two penalties allows to avoid over-fitting. The role of the two penalties is different, the $l_{1}$ term (sum of absolute values) enforces the solution to be sparse, the $l_{2}$ term (sum of the squares) preserves correlation among the variables. The training for selection and classification requires the choice of the regularization parameters for both $l_{1} l_{2}$ regularization and regularized least squares (RLS) denoted with $\tau^{*}$ and $\lambda^{*}$, respectively. In fact model selection and statistical significance is performed within two nested K-cross validation loops as in $[29,30]$. Being interested in a comprehensive list of relevant variables we fixed our attention on the lists obtained with the highest values for the correlation parameter $\mu$.

The statistical framework described above provides a set of $\mathrm{K}$ lists of selected variables, therefore it is necessary to choose an appropriate criterion [31] in order to assess a common list of relevant variables (probe-sets or proteins, in our case). We based ours on the absolute frequency, i.e. we decided to promote as relevant variables the most stable probe-sets across the lists. The threshold we used to select the final lists was chosen according to the slope variation of the number of selected genes vs. frequency (plot not shown), its value being $70 \%$. In this way we manage to cut out those variables that are not stable across the cross-validation lists, similarly to the procedure adopted in [30]. We also visualized the signatures in heat-map plots and $3 \mathrm{~d}$ visualizations of classified samples.

\section{Functional characterization of the gene signature}

Multiple probe-sets for a gene were collapsed to one entry per gene, based on the best frequency score. Non-mapping or non-coding probe-sets were discarded. The National Institute of Health Database for Annotation, Visualization and Integrated Discovery (DAVID) web-tool [32] was used to identify structural, functional, and pathway categories in the selected list. The analysis also ranked in detail the Gene Ontology (GO) [33] terms in the Biological Process (BP) domain including the identified probe-sets. The functional annotation was performed using the Expression Analysis Systematic Explorer (EASE) [34] with structural and functional class data from the GO, GenBank and UniGene databases, and with pathway data from Gene Map Annotator and Pathway Profiler (GenMAPP) [35], the Kyoto Encyclopedia of Genes and Genomes (KEGG) [36] and the Biocarta [37] databases. The Exploratory Gene Association Networks (EGAN) Java desktop application was also used to visualize the interactions among the selected genes [38].

\section{Real-time quantitative reverse transcription-PCR}

Following the same criteria for the case selection, we chose an additional set of patients (dataset 2), composed by 14 PAs and 4 mixed glial-neuronal tumours, in order 
to confirm and validate with $\mathrm{qPCR}$ the most significant genetic signatures emerging from gene-chip analysis. Each systems were in-house designed by a fine tuning procedure as described [39]. Specific primers were developed targeting: $A B B A 1$ [NM 138383], APOD [NM 001647], ARX [NM 139058], CXCL14 [NM 004887], FOSB [NM 001114171], FOXG1 [NM 005249], GPR17 [NM 001161415], LHX2 [NM 004789], NRXN2 [NM 015080], PTGD2S [NM 000954], SDC3 [NM 014654], SNX22 [NM 024798], SPOCK1 [NM 004598], TIMP4 [NM 003256] and ZFHX4 [NM 024721]. Primers sequences and the amplification conditions are reported in Additional file 2. Beta actin $(A C T B)$ [NM 001101], Pyruvate kinase (PKM2) [NM 002654] and Beta-2-microglobulin (B2M) [NM 004048] were used as the endogenous control genes for each tumour specimen [39]. Amplifications were performed using an ABI PRISM 7500 HT Sequence Detection System (Applied Biosystems, Foster City, CA) and primer concentrations were adjusted accordingly to the assays temperature. Validation of each system was performed using standard curves (SCs) on cDNA derived from the 1603-MED medulloblastoma cell line [40].

The reproducibility of the calibration curve was analyzed qPCR efficiencies of each system were calculated as described [41]. The relative quantification of genes transcript was performed according to the comparative method $\left(2^{-\Delta \mathrm{Ct}}\right)$, Applied Biosystems User Bulletin no. 2P/ $N$ 4303859) [42,43], using the value emerged by geometric mean of B2M, PKM2 and $A C T B$ as the normalizer $\left(\mathrm{Ct}_{r e f}\right)$. Gene expression levels of the 18 candidates were calculated for each LGG sample by the $2^{-\Delta \Delta C t}$ equation using as $\Delta \mathrm{Ct}_{\text {ref }}$ the median $\Delta \mathrm{Ct}$ value among all cases. The Minimum Information for Publication of qPCR Experiments (MIQE) are provided [44].

\section{Statistical validation}

Comparisons of the quantitative data of gene expressions were performed by the Mann-Whitney $U$ test since the normality and homoscedasticity assumptions were not fulfilled. Statistical tests were 2-sided, and a p-value less than 0.05 was considered statistically significant.

We also performed a multivariate data analysis by employing the algorithm known as Regularized Least Squares (RLS) [45]. The algorithm is based on the minimization of a functional depending on a least square error term combined with a regularization term, i.e., the $\mathrm{l}_{2}$ term (sum of the squares). Similarly to the $l_{1} l_{2}$ algorithm, RLS is run in a double nested cross-validation framework to avoid selection bias.

\section{Results}

Biologically validated molecular fingerprint of infratentorial versus supratentorial LGGs

We conducted a high-resolution analysis of genome-wide expression patterns on 40 paediatric LGGs, including 17 arising in infratentorial and 23 in supratentorial regions (Table 1), using Affymetrix HG-U133 Plus 2.0 chip-arrays. To select a list of highly discriminative probe-sets, we applied the $l_{1} l_{2}$ selection statistical framework to the dataset. The system performance was evaluated by its corresponding cross-validation error, as low as $8 \%$. The resulting list, reported in Additional file 3, consists of 331 probe-sets, sorted according to their frequency score and corresponding to the maximum value of the correlation parameter $\mu$.

The strong discriminative power of the selected probe-sets is depicted by either a heat-map plot or a multivariate representation (Figure 2a,b). The FA case was not classified neither supratentorial nor infratentorial by the $l_{1} l_{2}$ algorithm, showing the robustness of the method.

In order to obtain a $3 \mathrm{D}$ visualization, the expression data restricted to the 331 probe-sets was projected on its first 3 principal components, i.e., the components of maximum variance. It is evident that the two classes are clearly separated in the multidimensional space (Figure 2b). In Table 2 we list the selected genes and the highest frequency score associated to each of them.

Over-representation analysis using DAVID web-tool revealed that the main $\mathrm{GO}$ terms in the biological process (BP) domain include: neuronal development, brain morphogenesis and anatomical structure development. Thanks to the EGAN software program, that interfaces with existing GO and literature annotation of the genes and with canonical pathways to perform enrichment statistics, molecular networks based on direct or indirect gene-gene interactions were created for the list of 206 genes we identified. The most enriched pathways are chemokine signaling, mitogen-activated protein kinase (MAPK) signaling, $\mathrm{T}$ cell receptor signaling and cell adhesion molecules (CAMs) pathways (Figure 2c).

Using the available WEB-based gene set analysis tools, a functionally based criterion was then applied to the list of 206 genes in order to select groups of genes that were most represented in the tumour development pathways and that were top-ranked in the $l_{1} l_{2}$ list. The resultant minimal list was composed by 19 out of 331 probe-sets, corresponding to 15 loci, see Table 3 . Relative functional analysis showed that the selected genes enriched BP related to CNS neuron differentiation, forebrain development, regulation of metabolic process, and cell proliferation. A brief comment of each locus is reported in Additional file 4, listing the main protein functions for the 15 genes that significantly discriminate infratentorial versus supratentorial LGGs.

\section{qPCR analysis}

In order to confirm and validate the results of microarray analyses, we considered 52 samples measured with qPCR, whose 34 samples from dataset 1 subjected to microarray experiments and 18 samples from dataset 2, on which 


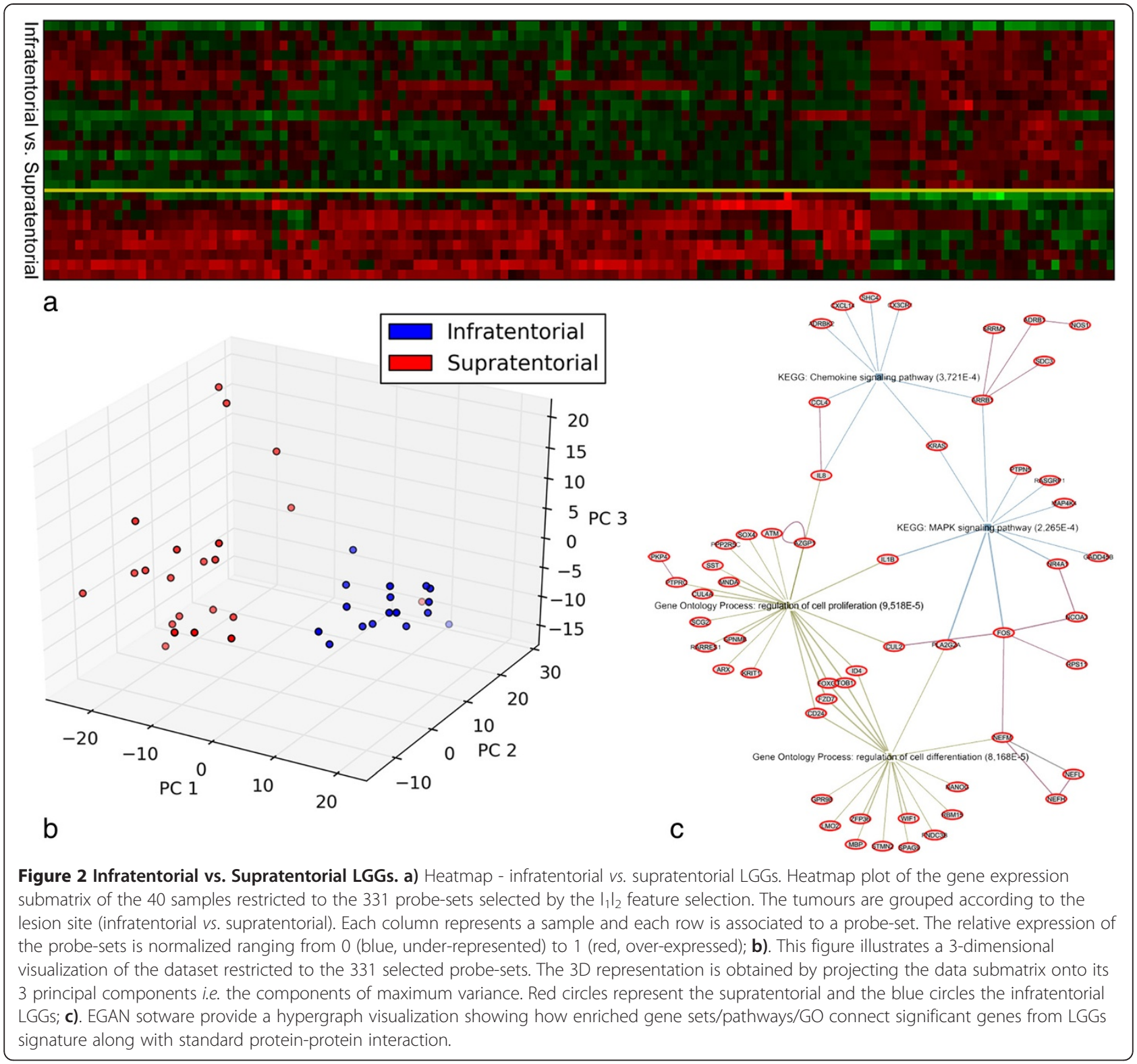

only qPCR was performed (see Figure 1). The relative quantification of the gene expression level for each gene was performed according to the comparative method $2^{-\Delta \Delta C t}$, using the averaged $\Delta \mathrm{Ct}$ value on all the LGG samples as tissue control $\left(\Delta \mathrm{Ct}_{r e f}\right)$. All 15 loci (represented by 19 probe-sets) were confirmed and validated (Table 4). The qPCR confirmed that all the 15 genes were differentially expressed between infratentorial versus supratentorial LGGs in multivariate analysis (RLS) (Table 4). Indeed, the Mann-Whitney test identified 5 out of 15 genes which were also significant in univariate analysis (Table 4). They were: aristaless related homeobox $(A R X)$, chemokine (C-X-C motif) ligand 14 (CXCL14), G protein-coupled receptor 17 (GPR17), LIM homeobox 2 (LHX2) and prostaglandin D2 synthase (PTGD2S), whose expressions resulted down-regulated in infratentorial tumours and up-regulated in supratentorial ones. For each sample we measured the qPCR expressions of the 5 selected genes and we verified that the expressions were up- and down- regulated according to the lesion site (Figure 3a).

Next, to build a multivariate statistical model on the qPCR data and validate the results of the microarray analyses we applied RLS classification method. The LOO-cross-validation error was $25 \%$. The multivariate model obtained with the RLS analysis was used to classify the available samples, achieving an accuracy of $91 \%$. The classification results were compared to 
Table $\mathbf{2}$ Infratentorial vs Supratentorial LGGs: selected genes by $I_{1} I_{2}$

\begin{tabular}{|c|c|c|c|c|c|c|c|c|c|}
\hline Gene & f.s. & Gene & f.s. & Gene & f.s. & Gene & f.s. & Gene & f.s. \\
\hline $\mathrm{ABBA} 1$ & 100 & KIAA2022 & 100 & KIAA1189 & 98 & $\mathrm{RHOB}$ & 92 & ZNF228 & 78 \\
\hline ADRBK2 & 100 & NRGN & 100 & KLHDC8A & 98 & RORB & 92 & LTB4DH & 75 \\
\hline ALK & 100 & PAX3 & 100 & LRAP & 98 & SCG2 & 92 & TMEPAI & 75 \\
\hline AMMECR1 & 100 & $\mathrm{PCDH} 8$ & 100 & MAN2A1 & 98 & SLC10A4 & 92 & BMS1P5 & 72 \\
\hline ANKRD10 & 100 & PCDHB16 & 100 & $\mathrm{NEFH}$ & 98 & SNRPN & 92 & KIAA1967 & 72 \\
\hline APOD & 100 & PCNX & 100 & NHSL1 & 98 & SOX4 & 92 & KRAS & 72 \\
\hline ARRB1 & 100 & PDGFRA & 100 & NOS1 & 98 & SPRN & 92 & MNDA & 72 \\
\hline ARX & 100 & PKIB & 100 & NR4A1 & 98 & TOB1 & 92 & CUL4A & 70 \\
\hline ATP6V0E1 & 100 & PPM1E & 100 & PLK2 & 98 & AKAP11 & 90 & NRXN2 & 70 \\
\hline ATXN3 & 100 & PPP1R9A & 100 & POLR2J2 & 98 & FCF1 & 90 & VSNL1 & 70 \\
\hline BICD1 & 100 & PRO1073 & 100 & POSTN & 98 & GFAP & 90 & & \\
\hline RBM15 & 100 & RARRES1 & 100 & PSD3 & 98 & GPR98 & 90 & & \\
\hline C1QL1 & 100 & RASGRP1 & 100 & RPS11 & 98 & MAP4K4 & 90 & & \\
\hline CAPN3 & 100 & RCOR1 & 100 & SPAG9 & 98 & RAB27B & 90 & & \\
\hline CCDC76 & 100 & RGS4 & 100 & SPATA18 & 98 & $\mathrm{SHC} 4$ & 90 & & \\
\hline CD24 & 100 & RRM2 & 100 & SPOCK1 & 98 & VAMP1 & 90 & & \\
\hline CHAD & 100 & SDC3 & 100 & STON2 & 98 & CRYAB & 88 & & \\
\hline $\mathrm{CNIH} 3$ & 100 & SHROOM3 & 100 & TMEM158 & 98 & DOCK9 & 88 & & \\
\hline $\mathrm{COCH}$ & 100 & SLC35A3 & 100 & TSPAN5 & 98 & FAM149A & 88 & & \\
\hline COG5 & 100 & SMA4 & 100 & ZC3H7A & 98 & FNDC3B & 88 & & \\
\hline CREB5 & 100 & SMOC1 & 100 & ADARB1 & 95 & FOSB & 88 & & \\
\hline CTGLF1 & 100 & SNX21 & 100 & CAMK2N1 & 95 & N4BP2 & 88 & & \\
\hline CX3CR1 & 100 & SOX10 & 100 & CAMKK2 & 95 & NUCKS1 & 88 & & \\
\hline CXCL14 & 100 & STMN2 & 100 & CDC2L5 & 95 & RPL37A & 88 & & \\
\hline CYR61 & 100 & STXBP6 & 100 & COL22A1 & 95 & SST & 88 & & \\
\hline ENC1 & 100 & SUSD5 & 100 & GLTSCR2 & 95 & ZNF226 & 88 & & \\
\hline EPHX1 & 100 & TIMP4 & 100 & GPNMB & 95 & GRM3 & 85 & & \\
\hline F2RL1 & 100 & TMTC4 & 100 & GRIA4 & 95 & KIAA1919 & 85 & & \\
\hline FBXL3 & 100 & TNFAIP6 & 100 & LRRFIP1 & 95 & PGM2L1 & 85 & & \\
\hline FNDC1 & 100 & TRPM3 & 100 & LYZ & 95 & PTPRC & 85 & & \\
\hline FOS & 100 & U2AF1 & 100 & NANOG & 95 & CCL4 & 82 & & \\
\hline FOXG1 & 100 & ZFHX4 & 100 & OLFM2 & 95 & CUL2 & 82 & & \\
\hline FZD7 & 100 & ZFP36 & 100 & OPA1 & 95 & FBXL11 & 82 & & \\
\hline GADD45B & 100 & ZNF207 & 100 & PHCA & 95 & GARNL4 & 82 & & \\
\hline GNL3L & 100 & ZNF294 & 100 & PKP4 & 95 & LCMT2 & 82 & & \\
\hline GUCY1A3 & 100 & ADRB1 & 98 & PLEKHA2 & 95 & TMEM132E & 82 & & \\
\hline HINT3 & 100 & ALDOC & 98 & PMP2 & 95 & TYMS & 82 & & \\
\hline HS3ST3B1 & 100 & ANKRD22 & 98 & PPP2R5C & 95 & GGNBP2 & 80 & & \\
\hline ID4 & 100 & ATM & 98 & WIF1 & 95 & IL8 & 80 & & \\
\hline IRX2 & 100 & CCDC91 & 98 & ASCL1 & 92 & PLA2G2A & 80 & & \\
\hline KIAA0101 & 100 & CLEC4A & 98 & AZGP1 & 92 & PMS2L5 & 80 & & \\
\hline KRIT1 & 100 & COL9A2 & 98 & F11R & 92 & S100A1 & 80 & & \\
\hline LHX2 & 100 & ENPP2 & 98 & FZD8 & 92 & SEZ6L & 80 & & \\
\hline
\end{tabular}


Table 2 Infratentorial vs Supratentorial LGGs: selected genes by $\mathbf{l}_{\mathbf{1}} \mathbf{l}_{\mathbf{2}}$ (Continued)

\begin{tabular}{|c|c|c|c|c|c|c|c|}
\hline MBP & 100 & FAM107B & 98 & LCAT & 92 & ZNF423 & 80 \\
\hline MICAL2 & 100 & FAM89A & 98 & LMO2 & 92 & EPHA5 & 78 \\
\hline NAIP & 100 & FRMD4A & 98 & MIAT & 92 & GPBP1L1 & 78 \\
\hline NCOA3 & 100 & GALNT13 & 98 & NEFM & 92 & MCTP1 & 78 \\
\hline NEFL & 100 & GAS7 & 98 & PPP1CB & 92 & PTPN5 & 78 \\
\hline NR2E1 & 100 & GPR17 & 98 & PTGD2S & 92 & STMN4 & 78 \\
\hline
\end{tabular}

List of 206 gene symbols selected by the $I_{1} l_{2}$ procedure. For each Gene ID we report the highest frequency score. We share 14 genes, showed in bold, with the results reported by previous studies $[9,19,20]$.

those achieved by the multivariate model from the $l_{1} l_{2}$ analysis on the microarray data (Figure 3b). Needless to say, we could only compare the results on the samples in dataset 1 measured both with microarray and qPCR $(\mathrm{n}=34)$. 29 out of $34(85 \%)$ were correctly classified by both methods. The microarray and qPCR analyses could not correctly classify 3 cases, two of which were assigned to the right class by the qPCR model. One sample was incorrectly classified by both approaches hence 33 out of 34 were associated to the right class by either method.

\section{Microarray-based differences of infratentorial versus supratentorial PAs}

Similarly, we conducted the analysis only on 27 PAs out of 40 LGGs (the FA was excluded), whose 17 arising in infratentorial and 10 in supratentorial regions, see Table 1 . Thank to the application of the $l_{1} l_{2}$ selection framework to the dataset, a list of 136 highly discriminative probe-sets corresponding to 82 genes was selected (see Additional file 5). The system performance was evaluated by its corresponding cross-validation error, as low as $15.4 \%$.

The strong discriminative power of the 136 selected probe-sets is visualized in Figure 4a,b. As shown in Figure $4 \mathrm{~b}$, the two classes of PA related to site of lesion are clearly separated in the multidimensional space. Again, the functional characterization of the gene signature performed with different web-tools, shows distinct processes enriched, as following: nervous system development, cell morphogenesis and cell adhesion, MAPK cascade, and chemotaxis. Moreover, the main pathways coming out are: chemokine signaling, transforming growth factor beta (TGF- $\beta$ ) signaling, MAPK signaling, Glioma, and WNT signaling pathways. The gene signature of this question is almost completely included in the larger LGG gene signature (62 common genes), but nineteen genes were specifically related to PA histotype as a group (see genes reported in bold in Additional file 5). Intriguingly, gene ontology analysis showed that distinct genes among the 19 related to PA, create a network within the TGF- $\beta$ signaling pathway [KEGG ID: 04350].
Microarray-based differences of supratentorial tumours: mixed glial-neuronal tumours versus PAs

Finally, the same analysis pipeline was applied to 22 supratentorial LGGs to distinguish mixed glial-neuronal tumours (12 samples) from PAs (10 samples), see Table 1. The $l_{1} l_{2}$ algorithm selected a list of 103 highly discriminative probe-sets corresponding to 70 genes as shown in Additional file 6. Even in this case, the system performance with its corresponding cross-validation error, i.e., $27 \%$ was analyzed.

The list of 70 genes, able to discriminate mixed glial-neuronal tumours vs. PAs (Figure 4c,d), includes genes involved in the extracellular matrix organization, forebrain development, and neuron differentiation such as distal-less homeobox 1 and 2 ( $D L X 1, D L X 2)$, immune response, such as hemoglobin alpha 1 and 2 (HBA1/2), chemokine (C-X-C motif) ligand 12 (CXCL12), chemokine (C-C motif) ligand 5 (CCL5), and metabolic proteins (Additional file 4). Distinct pathways are enriched: toll-like receptor signaling, focal adhesion, extracellular matrix constituents and remodeling machinery, and extracellular matrix (ECM) receptor interaction pathways. Interestingly, the presence of gene family of collagen such as collagen type I, alpha 1 and 2 (COL1A1, COL1A2), collagen type III, alpha1 (COL3A1), collagen type V, alpha 1 (COL5A1), and collagen type VI, alpha 2 and 3 (COL6A2, COL6A3) are significantly represented in mixed glial-neuronal tumours.

\section{Discussion}

A major emphasis has historically been placed on stratifying LGGs diagnosis or therapy on the basis of pathological and molecular genetic criteria. However, the increasing application of molecular approaches is transforming the way to categorize these tumours, since it seems that histologically comparable lesions may exhibit diverse patterns of gene expression and genomic alterations $[5-7,9,19,20]$. This investigation has focused on the identification of a specific gene signature based on high-throughput techniques that provide a genome-wide snapshot of LGGs with respect to both distinct lesion site in the brain and histotype. 
Table 3 Molecular fingerprint of LGGs composed by the selected 15 genes

\begin{tabular}{ll}
\hline Gene/Locus & Description \\
\hline FOXG1 / 14q13 & forkhead box G1 \\
& \\
GPR17 / 2q21 & \\
CXCL14 / 5q31 & G protein-coupled receptor 17 \\
ARX / Xp21 & chemokine (C-X-C motif) \\
ligand 14 \\
aristaless related homeobox
\end{tabular}
Biological Process

GO:0007417 CNS development GO:0022008 neurogenesis

GO:0030900 forebrain development GO:0048699 generation of neurons GO:0021954 CNS neuron development GO:0021953 CNS neuron differentiation GO:0048666 neuron development GO:0007423 sensory organ development GO:0043583 ear development GO:0009953 dorsal/ventral pattern formation GO:0006355 regulation of transcription, DNA-dependent GO:0051252 regulation of RNA metabolic process GO:0045449 regulation of transcription GO:0019219 regulation of nucleobase, nucleoside, nucleotide and nucleic acid GO:0048667 cell morphogenesis involved in neuron differentiation GO:0048812 neuron projection morphogenesis GO:0048858 cell projection morphogenesis GO:0032990 cell part morphogenesis GO:0031175 neuron projection development GO:0007165 signal trasduction GO:0007186 G-protein coupled receptor protein signaling pathway GO:0006995 immune response GO: 0006935 chemotaxis GO:0007267 cell-cell signaling GO:0007165 signal trasduction GO:0007417 CNS development, GO:0022008 neurogenesis GO:0030900 forebrain development, GO:0048699 generation of neurons GO:0021954 CNS neuron development, GO:0021987 cerebral cortex development GO:0021543 pallium development

GO:0006355 regulation of transcription, DNA-dependent GO:0051252 regulation of RNA metabolic process

GO:0045449 regulation of transcription

GO:0019219 regulation of nucleobase, nucleoside, nucleotide and nucleic acid GO:0048667 cell morphogenesis involved in neuron differentiation GO:0048812 neuron projection morphogenesis GO:0048858 cell projection morphogenesis GO:0032990 cell part morphogenesis GO:0031175 neuron projection development

GO:0007417 CNS development GO:0022008 neurogenesis

GO:0030900 forebrain development GO:0048699 generation of neurons GO:0048666 neuron development GO:0009953 dorsal/ventral pattern formation GO:0006355 regulation of transcription, DNA-dependent GO:0051252 regulation of RNA metabolic process GO:0045449 regulation of transcription GO:0019219 regulation of nucleobase, nucleoside, nucleotide and nucleic acid GO:0048667 cell morphogenesis involved in neuron differentiation

GO:0048812 neuron projection morphogenesis GO:0048858 cell projection morphogenesis GO:0032990 cell part morphogenesis, GO:0031175 neuron projection development 
Table 3 Molecular fingerprint of LGGs composed by the selected 15 genes (Continued)

\begin{tabular}{|c|c|c|}
\hline APOD / 3q26.2qter & apolipoprotein D & G0.0006629 lipid metabolic process \\
\hline \multirow[t]{2}{*}{ PTGD2S / 9q34.2-34.3 } & prostaglandin D2 synthase & GO:0006633 fatty acid biosynthetic process \\
\hline & 21 kDa (brain) & GO:0006810 transport \\
\hline SDC3 / 1 pter-p22.3 & syndecan 3 & GO:0007155 cell adhesion \\
\hline \multirow[t]{2}{*}{ NRXN2 / 11q13 } & neurexin 2 & GO:0007268 synaptic trasmission GO:0007269 neurotransmitter secretion \\
\hline & & GO:0007416 synapse assembly GO:0007155 cell adhesion \\
\hline $\mathrm{SNX} 22 / 15 \mathrm{q} 22.31$ & sorting nexin 22 & GO:0007165 signal trasduction GO:0007154 cell comunication \\
\hline \multirow[t]{4}{*}{ ZFHX4 / 8q21.11 } & zinc finger homeobox 4 & GO:0006355 regulation of transcription, DNA-dependent \\
\hline & & GO:0051252 regulation of RNA metabolic process \\
\hline & & GO:0045449 regulation of transcription \\
\hline & & GO:0019219 regulation of nucleobase, nucleoside, nucleotide and nucleic acid \\
\hline \multirow[t]{2}{*}{ SPOCK1 / 5q31 } & testican 1 & GO:0007417 CNS development GO:0007165 signal trasduction \\
\hline & & GO:0007155 cell adhesion \\
\hline ABBA1 / 16q22.1 & metastasis suppressor 1 like & GO:0007165 signal trasduction \\
\hline \multirow[t]{4}{*}{ FOSB / 19q13.32 } & FBJ murine osteosarcoma & GO:0006355 regulation of transcription, DNA-dependent \\
\hline & viral oncogene homolog B & GO:0051252 regulation of RNA metabolic process \\
\hline & & GO:0045449 regulation of transcription \\
\hline & & GO:0019219 regulation of nucleobase, nucleoside, nucleotide and nucleic acid \\
\hline
\end{tabular}

Gene annotations and GO Biological Process terms for the minimal list of 15 genes, selected for the biologically validated molecular fingerprint of LGGs related to site of lesion (infratentorial vs supratentorial).

Although an abundance of data is available on gene expression profiles of LGGs, they are often conflicting. Indeed, statistical methods for evaluation and interpretation of microarray data are still evolving. We successfully adopted an analysis workflow (Figure 1) able to overcome a major criticality in high-throughput studies, that is to find robust, reproducible and biologically sound results $[16,46]$. Details of the workflow description are reported in Additional file 1.

\section{Brain region-specific gene signature among LGGs}

Question (1) was used to assess the procedure and represent the first example of biologically validated $l_{1} l_{2}$ framework with an independent methodology. Indeed, this query is the one with more samples available as well as the one already investigated in previous works $[9,19]$. The provided outcome from $l_{1} l_{2}$ was a list of 331 probe sets (Additional file 3), corresponding to 206 loci, above $70 \%$ of frequency. $l_{1} l_{2}$ produces a multi-gene model and only a multidimensional representation can correctly visualize its strong discriminative power (Figure $2 b$ ). The figure shows that the infratentorial tumours group is spatially separated from the supratentorial counterpart.

Our analysis identified various interesting genes which encode cell adhesion molecules, ECM, extracellular matrix, lipid metabolism, CNS development, cell differentiation, transcription regulation, and invasion-related proteins. Unlike Potter et al. reported [21], our results are in line with previous findings that clearly defined the existence of PA subgroups. Indeed, 14 out of 206 differentially expressed genes (reported in bold in Table 2) were reported by previous studies $[9,19,20]$. Wong and colleagues identified two subgroups of PA reporting a list of significant differentially expressed genes involved in cell adhesion, regulation of cell growth, cell motility, and angiogenesis [19]. Sharma and colleagues reported differential expression of genes playing a role in forebrain development as LHX2 and nuclear receptor subfamily 2, group $\mathrm{E}$, member 1 (NR2E1), and hindbrain development as paired box gene 3 (PAX3) and iroquois homeobox protein 2 (IRX2), able to stratify infratentorial from supratentorial PAs [9]. The comparison with the Sharma's data, the only comparable, inasmuch as homologous for case selection, sample processing and Affymetrix platform, allowed us, even using our own statistical approach, to identify five genes (LHX2, NR2E1, PAX3, IRX2, and zinc finger homeobox 4, (ZFHX4) common to both analyses.

To investigate paediatric LGG development related to site of lesion (infratentorial vs. supratentorial) [47], we next proceeded by selecting those candidate genes that were most represented among all the high-ranked pathways for the validation process by using our in-house designed qPCR systems on 52 samples (34 samples belonged to dataset 1, while 18 samples were from dataset 2). Finally, the list of candidates comprised 19 probe-sets corresponding to 15 loci in total (Table 3). We validated 
Table 4 qPCR expression values for the selected 15 genes

\begin{tabular}{|c|c|c|c|c|c|c|}
\hline \multirow[t]{2}{*}{ Gene name } & \multicolumn{2}{|c|}{ Supratentorial } & \multicolumn{2}{|c|}{ Infratentorial } & \multirow[t]{2}{*}{$\Delta C \mathrm{t}$ median } & \multirow{2}{*}{$\begin{array}{l}\text { I. vs. S. } \\
\text { p-value }\end{array}$} \\
\hline & $\Delta \mathrm{Ct}$ & $2^{-\Delta \Delta C t}$ & $\Delta \mathrm{Ct}$ & $2^{-\Delta \Delta C t}$ & & \\
\hline ABBA 1 & 2.19 & 1.46 & 2.88 & 0.9 & 2.72 & - \\
\hline APOD & -1.33 & 0.69 & -2.46 & 1.6 & -1.87 & - \\
\hline ARX & 6.03 & 2.46 & 8.25 & 0.53 & 7.33 & 0.03 \\
\hline CXCL14 & 0.84 & 12.61 & 7.17 & 0.18 & 4.5 & 0.009 \\
\hline FOSB & 4.15 & 0.98 & 3.84 & 1.27 & 4.11 & - \\
\hline FOXG1 & 4.65 & 0.7 & 3.38 & 1.69 & 4.14 & - \\
\hline GPR17 & 2.85 & 1.52 & 8.18 & 0.04 & 3.43 & 0.0049 \\
\hline LHX2 & 4.6 & 7.59 & 9.04 & 0.35 & 7.52 & 0.01 \\
\hline NRXN2 & 6.28 & 1.18 & 6.52 & 0.99 & 6.51 & - \\
\hline PTGD2S & 1.34 & 1.66 & 5.4 & 0.1 & 2.08 & 0.0049 \\
\hline SDC3 & 2.9 & 0.94 & 1.79 & 2.03 & 2.81 & - \\
\hline SNX22 & 6.98 & 0.73 & 6.34 & 1.11 & 6.5 & - \\
\hline SPOCK1 & 3.99 & 0.88 & 3.06 & 1.69 & 3.81 & - \\
\hline TIMP4 & 2.62 & 0.92 & 2.42 & 1.05 & 2.48 & - \\
\hline ZFHX4 & -0.43 & 0.85 & -0.84 & 1 & -0.84 & - \\
\hline
\end{tabular}

qPCR values for dataset 1 (34 samples) and 2 (18 samples). Gene expressions were significantly different for all listed genes by multivariate analysis (RLS). The genes in bold are those passing the Mann-Whitney test (only its significant $\mathrm{p}$-values are reported on the right column). S: Supratentorial, I: Infratentorial.

the generalization ability of the 15 gene signature by applying a multivariate statistical model on the qPCR data of dataset 1 (34 samples). Such multivariate model, obtained with a RLS analysis, was used to assign the samples to a group and the classification results were compared to the $l_{1} l_{2}$ microarray-based model (Figure $3 \mathrm{~b}$ ).
The two independent methods have good performances, being able to associate 33 out of 34 samples to the right class. Moreover, 5 out of 15 genes emerged from the univariate Mann-Whitney test on the qPCR data, confirming and enhancing the LGG differences in infratentorial as compared with supratentorial regions, see Table 4 and Figure 3a. As shown in Figure 3a, a group of 4 genes (ARX, GPR17, LHX2 and CXCL14) well stratified LGGs between infratentorial and supratentorial tumours. $A R X$ is a homeobox-containing gene expressed during development. This gene is involved in CNS development and in cell proliferation in forebrain [GO:0021846]. Mutations in this gene cause $\mathrm{X}$-linked mental retardation and epilepsy. To the best of our knowledge, $A R X$ was never associated with LGGs. GPR17 is a G-protein involved in signal transduction [GO:0007165]. LHX2 is downregulated in infratentorial tumours as already reported [9]. CXCL14 is a chemokine associated with tumour development [GO:0006995], and PTDG2S whose functions are associated to lipid metabolism [GO:0006633], might be involved in controlling the proliferation rate of LGGs.

Additionally, the predominant terms related to pathways consisted of MAPK signaling pathway, containing at least 12 genes, followed by chemokine signaling pathway with 8 genes enriched. These findings reinforce the observations of several consecutive articles about aberrant activation of the mitogen-activated protein kinase (MAPK) pathway in LGGs [48]. The identification of a brain region-specific gene signature suggests that LGGs at different sites may be distinct in terms of biological properties and tumorigenesis despite the same histology. KIAA1549:
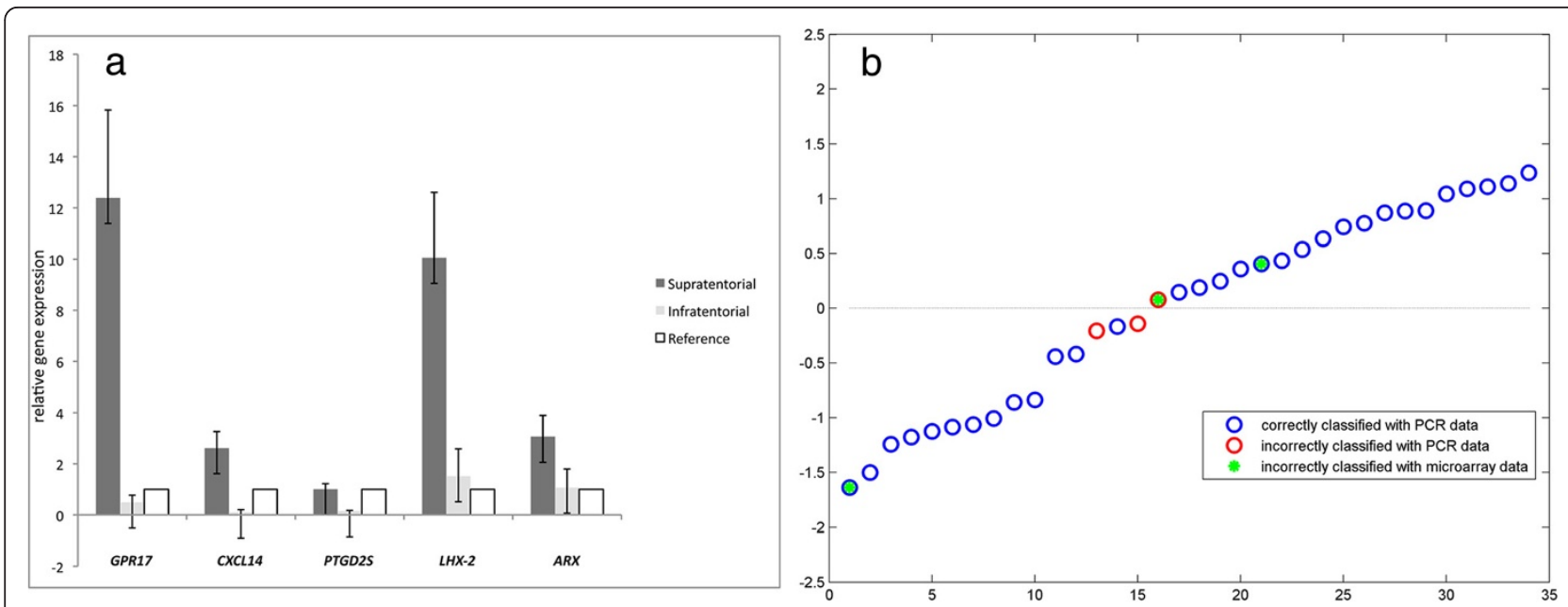

Figure 3 The best differentially expressed genes with qPCR between infratentorial and supratentorial LGGs. a) the best differentially expressed genes with qPCR between infratentorial and supratentorial LGGs. Relative gene expression for the best 5 differentially expressed genes selected with univariate Mann-Whitney test; $\mathbf{b}$ ) in this plot we show the comparison between the microarray and the values of the estimated RLS classification function for each sample measured by both qPCR and microarray. The negative values are assigned to the infratentorial site, while positives are the supratentorial ones. The blue circles correspond to the correctly classified cases by qPCR. The red circles indicate the misclassified samples by qPCR. The green dots are the misclassified samples by the microarray model. 


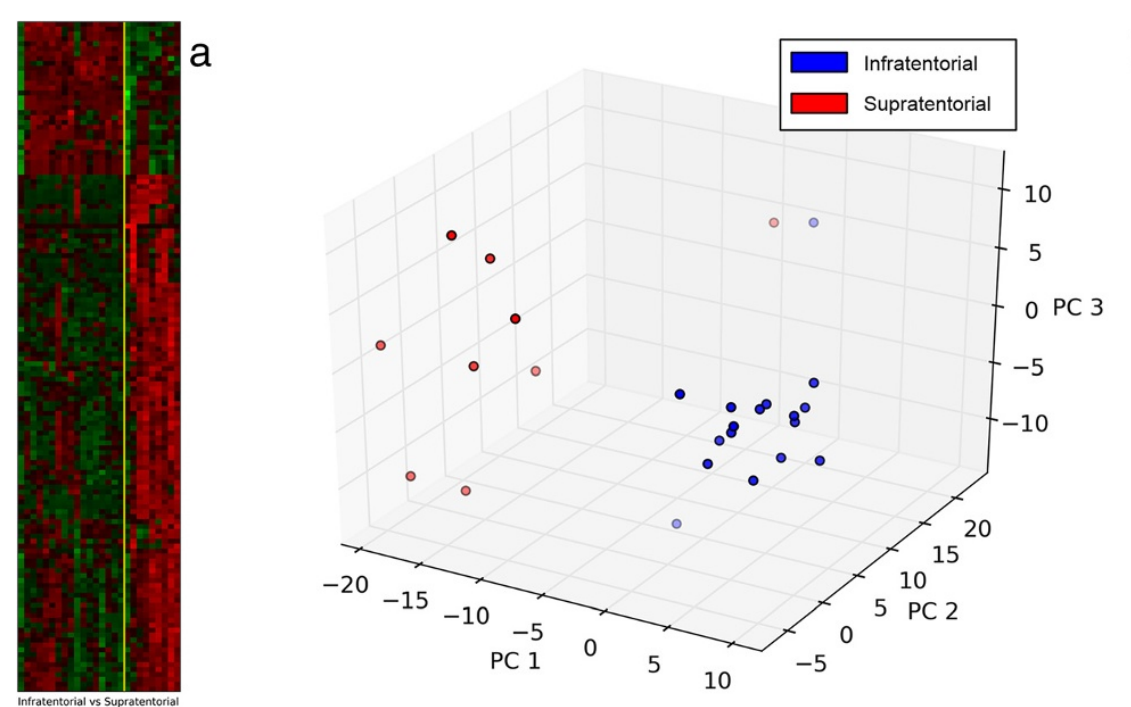

b

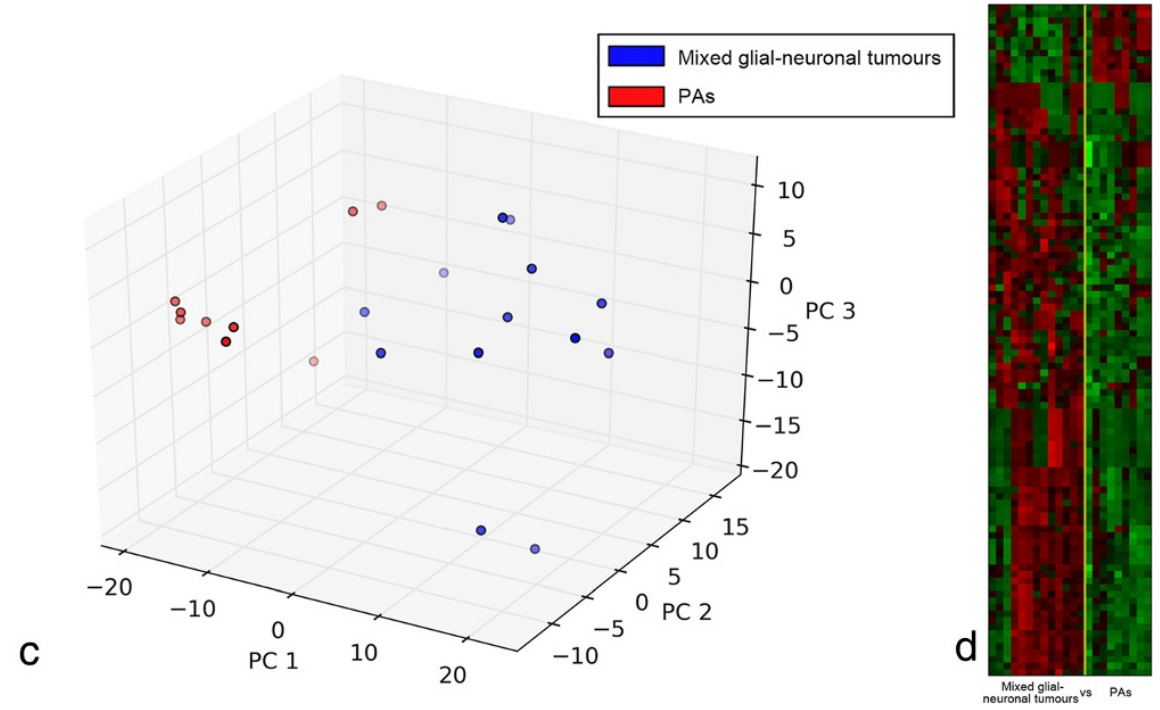

Figure 4 Molecular fingerprinting sub-classify infratentorial from supratentorial PAs as well as separate mixed glial-neuronal tumours from PAs. a) heatmap - infratentorial vs. supratentorial PAs. Heatmap plot of the gene expression submatrix of the 27 PAs restricted to the 136 probesets selected by the $I_{1} I_{2}$ feature selection. The tumours are grouped according to the lesion site (infratentorial vs. supratentorial). Each column represents a sample and each row is associated to a probe-set. The relative expression of the probe-sets is normalized ranging from 0 (blue, under-represented) to 1 (red, over-expressed), b) This figure illustrates a 3-dimensional visualization of the dataset restricted to the 136 selected probe-sets. The 3D representation is obtained by projecting the data submatrix onto its 3 principal components i.e. the components of maximum variance. Red circles represent the supratentorial and the blue circles the infratentorial PAs; $\mathbf{c}$ ) This figure illustrates a 3D projection of the dataset restricted to the 103 selected probe-sets for the supratentorial tumours: mixed glial-neuronal tumours vs. PAs. The 3D representation is obtained by projecting the data submatrix onto its 3 principal components i.e. the components of maximum variance. Red circles represent the PAs and the blue circles the mixed glial-neuronal tumours; d) Heatmap plot of the gene expression submatrix of the 22 supratentorial tumours restricted to the 103 probe-sets selected by the $l_{1} l_{2}$ feature selection. The tumours are grouped according to the histotype (mixed glial-neuronal tumours vs. PAs) Each column represents a sample and each row is associated to a probe-set. The relative expression of the probe-sets is normalized ranging from 0 (blue, under-represented) to 1 (red, over-expressed).

$B R A F$ fusions were analyzed in the LGG cohort and we found the gene fusion slightly more frequent in infratentorial (38.5\%) versus supratentorial (25\%) tumours, while we didn't note any difference for BRAF V600E mutation. Moreover, we did not identify significantly improved progression-free survival in tumours with gene-fusions or BRAF V600E mutation.

\section{Identification of a subgroup of 19 genes specifically related with PA histotype}

Next, to molecularly characterize PA able to distinguish infratentorial versus supratentorial, $l_{1} l_{2}$ analysis were conducted only on 27 PAs out of 37 LGGs, whose 17 arising in infratentorial and 10 in supratentorial regions, see Table 1. A gene signature of 82 genes 
(see Additional file 5) well distinguishes PA arising supratentorial versus infratentorial regions (Figures 4a,b). Significant biological processes represented include GO terms of nervous system development, cell morphogenesis, cell differentiation and cell adhesion, MAPKKK cascade, chemotaxis, and regulation of neurogenesis. We found that, together with $A R X$, forkhead box G1 (FOXG1) was strongly represented in PA. FOXG1 is an oncogenic transformer which could play an important role in controlling both cell proliferation and forebrain cell differentiation in PA [21,49-51].

Through the comparison of gene lists between LGG and PA, we found 19 genes specifically related with PA histotype as a group (genes in bold in Additional file 5). The functional analysis showed that several genes create a network within the (TGF- $\beta$ )-signaling pathway. This pathway possess a dual role in oncogenesis. In some tumour types, i.e., in high-grade gliomas, TGF-beta becomes an oncogenic factor, while it is also considered a tumour suppressor factor in normal epithelial cells and astrocytes. Moreover, noncanonical TGF-beta signaling pathways interact, through RSmads molecules, with MAPK signaling pathway [52]. Thanks to this interaction, it is likely to assume an active involvement of TGF-beta signaling pathway in the PA development.

Our analysis shows a strong difference between supratentorial and infratentorial PAs. In fact, cerebellar PAs, corresponding to the classical description of the biphasic tumour with compact areas with piloid cells and Rosenthal fibers and microcistic areas with granular eosinophilic bodies [3], seem to be defined by a specific gene signature versus supratentorial PAs. Therefore, this molecular fingerprint is able to better sub-classify such a morphologically heterogeneous tumours.

\section{Neurogenesis, cell motility and cell growth genes dichotomize mixed glial-neuronal tumours versus PAs}

Finally, the analysis on 22 supratentorial LGGs identified a list of 70 genes (see Additional file 6) able to dichotomize mixed glial-neuronal tumours versus PAs (Figure 4c,d). The signature consists of genes encoding adhesion, ECM-receptor interaction, matrix extracellular organization, neurogenesis, immune response, and metabolic proteins. Several genes are components of collagen gene family whose functions are associated with extracellular matrix (ECM) reorganization. Intriguingly, changes in expression of genes controlling neurogenesis (DLX1, DLX2), cell growth such as insulin-like growth factor 2 (IGF2), insulinlike growth factor binding protein 6 (IGFBP6) and latent transforming growth factor beta binding protein (LTBP2), cell motility such as 11 cell adhesion molecule (L1CAM), COL3A1 and integrin, alpha 8 (ITGA8), and interactions with the surrounding environment such as lumican (LUM), COL1A1, COL6A3 and periostin, osteoblast specific factor
(POSTN) appear to be linked to the presence of neuronal cell component.

Because of their rare occurrence, little is yet known about the molecular pathology of mixed glial-neuronal neoplasms and the cytogenetic and molecular genetic studies reported are very few [53-55]. Our findings show the complexity and vitality of these tumours, shedding some light on features such their richness in connective tissue and, they point to some interesting candidate genes (i.e., $D L X 1, D L X 2)$ worth further investigations that could help the pathologists in the differential diagnosis.

From a biological point of view, it is remarkable that the mixed glial-neuronal tumours are strikingly separated from PAs, allowing us to look differently at mixed glial-neuronal tumours in which, generally, the glial component catches the attention of the pathologists and contributes to grading. Our findings, indeed, shed some light on the biological complexity of the mixed glial-neuronal tumours, still poorly known. It remains to be established if mixed glial-neuronal tumours differ from PAs because of their ganglion-like component or because of their glial one or both. What seems indubitable is that the ganglion cell component is not a bystander. Future functional studies are needed to evaluate these targets in paediatric mixed glial-neuronal tumours versus PAs but evidence supports a role for these gene candidates in tumorigenesis.

\section{Conclusion}

The identification of a brain region-specific gene signature suggested that LGGs at different sites may be distinct in terms of biological properties and tumorigenesis. The success of our methodology carries implications for improving the diagnosis and possibly prognosis of LGGs. The method efficiently finds and ranks genes that can distinguish one histotype from another. In addition, we performed clustering and classification of GO categories and possibly altered pathways on the basis of gene expression in infratentorial versus supratentorial LGGs, in particular in the PAs, and among supratentorial tumours, in mixed glial-neuronal tumours versus PAs.

The analyses reinforce previous observations about aberrant activation of the mitogen-activated protein kinase (MAPK) pathway in LGGs but, still point to an active involvement of TGF- $\beta$ signaling pathway in the PA development and, emphasize some interesting candidate genes worth further investigations for the mixed glialneuronal tumours. Considering the high clinical and biological relevance of the disease, as these tumours are detrimental to children, and since the genetic background of paediatric glial tumours is still unsatisfied, this methodological work could mark the starting line. A genotypephenotype correlation of LGGs is instrumental to improve classification and differential diagnosis. Impact of molecular classification will likely change how LGGs are both 
diagnosed and treated henceforward. This paper thus provides a novel global view of the molecular differences between infratentorial and supratentorial LGGs.

Further investigation and validation by experiments should be targeted to the exploration of a deeper genotype-phenotype correlation in those LGG cases who undergo malignant transformation.

\section{Additional files}

Additional file 1: Workflow for high-throughput gene-expression studies. Description and discussion, including references, of the pipeline used for gene-expression studies.

Additional file 2: qPCR systems. Primers sequences and the amplification conditions of the 15 selected genes validated by $\mathrm{qPCR}$, including 3 genes used as the endogenous control for each tumour specimen.

Additional file 3: Selected probe-sets by $I_{1} I_{2}$ for LGG related to the site of lesion. List of 331 probe-sets selected by the $\left.\left.\right|_{1}\right|_{2}$ procedure for LGG related to the site of lesion (infratentorial versus supratentorial). For each probe-set we report the corresponding Gene ID and its frequency score (f.s.).

Additional file 4: Microarray-based differences of LGG related to site of lesion. A brief comment of each locus, listing the main protein functions and including references, for the 15 selected genes that significantly discriminate infratentorial versus supratentorial LGGs.

Additional file 5: Pilocytic Astrocytomas - infratentorial vs supratentorial, selected probe-sets by the $I_{1} l_{2}$ for PA related to the site of lesion. List of 136 probe-sets selected by $\left.\right|_{1} l_{2}$ for infratentorial vs supratentorial PAs. For each probe-set we report the Gene ID and its frequency score (f.s.). Genes reported in bold are those specific to PA histotype as a group.

Additional file 6: Supratentorial tumours: mixed glial-neuronal tumours vs. PAs, selected probe-sets by the $I_{1} l_{2}$. List of 103 probe-sets selected by the $\left.\left.\right|_{1}\right|_{2}$ procedure. For each probe-set we report the corresponding Gene ID and its frequency score (f.s.).

\section{Abbreviations}

PA: Pilocytic astrocytoma; WHO: World Health Organization; LGG: Low Grade Glioma; DIA: Desmoplastic infantile astrocytoma; DIG: Desmoplastic infantile ganglioglioma; GG: Ganglioglioma; FA: Diffuse fibrillary astrocytoma; CNS: Central nervous system; NF1: Neurofibromatosis type 1; RLS: Regularized least squares; DAVID: Database for annotation, visualization and integrated discovery; KEGG: Kyoto encyclopedia of genes and genomes; GO: Gene ontology; BP: Biological process; GenMAPP: Gene map annotator and pathway profiler; EASE: Expression analysis systematic explorer;

EGAN: Exploratory gene association network; MAPK: Mitogen-activated protein kinase; TGF- $\beta$ : Transforming growth factor-beta; CAM: Cell adhesion molecule; ECM: Extracellular matrix; ARX: Aristaless related homeobox; CXCL14: Chemokine (C-X-C motif) ligand 14; GPR17: G protein-coupled receptor 17; LHX2: LIM homeobox 2; NR2E1: Nuclear receptor subfamily 2, group E, member 1; PAX3: Paired box gene 3; IRX2: Iroquois homeobox protein 2; PTGD2S: Prostaglandin D2 synthase; ACTB: Beta actin; PKM2: Pyruvate kinase; B2M: Beta-2-microglobulin; DLX1/2: Distal-less homeobox 1, 2; HBA1/2: Hemoglobin alpha 1, 2; CXCL12: Chemokine (C-X-C motif) ligand 12; CCL5: Chemokine (C-C motif) ligand 5; COL1A1/2: Collagen type I, alpha 1, 2; COL3A1: Collagen type III, alpha1; COL5A1: Collagen type V, alpha 1; COL6A2/3: Collagen type VI, alpha 2, 3; FOXG1: Forkhead box G1; IGF2: Insulin-like growth factor 2; IGFBP6: Insulin-like growth factor binding protein 6; LTBP2: Latent transforming growth factor beta binding protein; L1CAM: L1 cell adhesion molecule; ITGA8: Integrin, alpha 8; LUM: Lumican; POSTN: Periostin, osteoblast specific factor; TIM4: TIMP metallopeptidase inhibitor 4; APOD: Apolipoprotein D; ZFHX4: Zinc finger homeobox 4; SDC3: Syndecan 3; NRXN2: Neurexin 2; SNX22: Sorting nexin 22; ABBA1: Metastasis suppressor 1 like; SPOCK1: Testican 1; FOSB: FBJ murine osteosarcoma viral oncogene homolog B; KIAA1549: KIAA1549; BRAF: v-raf murine sarcoma viral oncogene homolog B1.

\section{Competing interests}

The authors declare that they have no competing interests.

\section{Authors' contributions}

SM and AR performed the experiments, interpreted the data and wrote the manuscript. AB performed statistical data analysis, participated in guiding some experiments and wrote the manuscript. SM, CM, DF, GW and KN participated in performing statistical data analysis. PN carried out pathological data review, contributed to the study design and with RB, provided research and editorial assistance. $\mathrm{MH}$ and SP provided assistance for the clinical data. GM provided neuroradiological data. AC, GP and provided tissue samples from the surgery. MLG provided tissue samples from the tissue-bank and clinical data, and with VC contributed to the study design, collaborated to the research and review the manuscript. AV contributed to the statistical analyses design and review the manuscript. All authors read and approved the final manuscript.

\section{Acknowledgments}

This study was supported by the EU Integrated Project Health-e-Child IST2004-027749, by the Associazione Italiana per la Ricerca sui Tumori Cerebrali del Bambino (http://www.artuceba.org) and by Compagnia di San Paolo (Modelli e Metodi Computazionali Innovativi per la Biologia Molecolare in Ambito Oncologico). The authors would like to thank all the patients'families. The authors would also like to thank Salvatore Masecchia for his contribution to the experimental part, Lorenzo Rosasco for many useful discussions, Valerie Perricone for the editorial assistance, and Prof. David Ellison for the useful advice. We also thank Camusso Raffaella and Tedeschi Luca (Photographic Service, Giannina Gaslini Children's Research Hospital, Genoa, Italy) for their graphic assistance.

\section{Author details}

${ }^{1}$ Neurosurgery Unit, Istituto Giannina Gaslini, via G. Gaslini 5, 16147, Genoa, Italy. ${ }^{2} \mathrm{DISI}$ - Department of Computer Science, Università degli Studi di Genova, Via Dodecaneso, 35-16146, Genoa, Italy. ${ }^{3}$ Pathology Unit, Istituto Giannina Gaslini, via G. Gaslini 5, 16147, Genoa, Italy. ${ }^{4}$ Molecular Medicine Unit, Istituto Giannina Gaslini, via G. Gaslini 5, 16147, Genoa, Italy.

${ }^{5}$ Neuroradiology Unit, Istituto Giannina Gaslini, via G. Gaslini 5, 16147, Genoa, Italy. ${ }^{6}$ Siemens AG, Corporate Technology, Freyeslebenstr. 1, 91058, Erlangen, Germany. ${ }^{7} \mathrm{SCR}$ - Siemens Corporate Research, Princeton, NJ, USA.

${ }^{8}$ Neuro-oncology Unit, Istituto Giannina Gaslini, via G. Gaslini 5, 16147, Genoa, Italy.

Received: 4 April 2013 Accepted: 6 August 2013

Published: 15 August 2013

\section{References}

1. Sievert AJ, Fisher MJ: Pediatric low-grade gliomas. J Child Neurol 2009, 24(11):1397-1408.

2. Kaatsch P: Epidemiology of childhood cancer. Cancer Treat Rev 2010, 36:277-285

3. Louis DOH, Wiestler O, Cavenee W: World Health Organization Classification of Tumors of the Central Nervous System. Lyon: IARC 2007; 2007.

4. Ohgaki H, Kleihues P: Population-based studies on incidence, survival rates, and genetic alterations in astrocytic and oligodendroglial gliomas. J Neuropathol Exp Neurol 2005, 64:479-489.

5. Pollack IF, Biegel J, Yates A, Hamilton R, Finkelstein S: Risk assignment in childhood brain tumors: the emerging role of molecular and biologic classification. Curr Oncol Rep 2002, 4:114-122.

6. Faria C, Miguéns J, Antunes JL, Salgado D, Nunes S, et al: Pediatric brain tumors: genetics and clinical outcome. J Neurosurg Pediatr 2010, 5:263-270.

7. Hargrave D: Paediatric high and low grade glioma: the impact of tumour biology on current and future therapy. Br J Neurosurg 2009, 23:351-363.

8. Qaddoumi I, Sultan I, Broniscer A: Pediatric low-grade gliomas and the need for new options for therapy: Why and how? Cancer Biol Ther 2009, 8:4-10.

9. Sharma MK, Mansur DB, Reifenberger G, Perry A, Leonard JR, et al: Distinct genetic signatures among pilocytic astrocytomas relate to their brain region origin. Cancer Res 2007, 67:890-900.

10. Lee SH, Kim WT, Cornell-Bell AH, Sontheimer H: Astrocytes exhibit regional specificity in gap-junction coupling. Glia 1994, 11:315-325. 
11. Ruzicka BB, Fox CA, Thompson RC, Meng F, Watson SJ, et al: Primary astroglial cultures derived from several rat brain regions differentially express mu, delta and kappa opioid receptor mRNA. Brain Res Mol Brain Res 1995, 34:209-220.

12. Ducray F, Marie $Y$, Sanson M: Idh1 and idh2 mutations in gliomas. N Engl J Med 2009, 360:2248-2249.

13. MacDonald TJ, Pollack IF, Okada H, Bhattacharya S, Lyons-Weiler J: Progression-associated genes in astrocytoma identified by novel microarray gene expression data reanalysis. Methods Mol Biol 2007, 377:203-222.

14. Dupuy A, Simon RM: Critical review of published microarray studies for cancer outcome and guidelines on statistical analysis and reporting. J Natl Cancer Inst 2007, 99:147-157.

15. loannidis JPA: Why most published research findings are false. PLOS Med 2005, 2:e124.

16. Ioannidis JPA, Allison DB, Ball CA, Coulibaly I, Cui X, et al: Repeatability of published microarray gene expression analyses. Nat Genet 2009, 41:7.

17. Rickman DS, Tyagi R, Zhu XX, Bobek MP, Song S, et al: The gene for the axonal cell adhesion molecule tax- 1 is amplified and aberrantly expressed in malignant gliomas. Cancer Res 2001, 61:2162-2168.

18. Shai R, Shi T, Kremen TJ, Horvath S, Liau LM, et al: Gene expression profiling identifies molecular subtypes of gliomas. Oncogene 2003, 22:4918-4923.

19. Wong KK, Chang YM, Tsang YTM, Perlaky L, Su J, et al: Expression analysis of juvenile pilocytic astrocytomas by oligonucleotide microarray reveals two potential subgroups. Cancer Res 2005, 65:76-84.

20. Rorive S, Maris C, Debeir O, Sandras F, Vidaud M, et al: Exploring the distinctive biological characteristics of pilocytic and low-grade diffuse astrocytomas using microarray gene expression profiles. J Neuropathol Exp Neurol 2006, 65:794-807.

21. Potter N, Karakoula A, Phipps KP, Harkness W, Hayward R, et al: Genomic deletions correlate with underexpression of novel candidate genes at six loci in pediatric pilocytic astrocytoma. Neoplasia (New York, NY) 2008, 10:757-772.

22. Marko NF, Prayson RA, Barnett GH, Weil RJ: Integrated molecular analysis suggests a three-class model for low-grade gliomas: a proof-of-concept study. Genomics 2010, 95:16-24.

23. Irizarry R, Bolstad B, Collin F, Cope L, Hobbs B, et al: Summaries of affymetrix genechip probe level data. Nucleic Acids Res 2003, 31:e15.

24. Destrero A, De Mol C, Odone F, Verri A: A sparsity-enforcing method for learning face features. IEEE Trans Image Process 2009, 18(1):188-201.

25. Bengtsson H, Simpson K, Bullard J, Hansen K: Aroma.affymetrix: A generic framework in $\mathrm{R}$ for analyzing small to very large Affymetrix data sets in bounded memory. Tech Report \#745, Department of Statistics, University of California, Berkeley, February 2008

26. Zou H, Hastie T: Regularization and variable selection via the elastic net. J ROY STAT SOC B 2005.

27. Mol CD, Vito ED, Rosasco L: Elastic net regularization in learning theory. J Complex 2009.

28. De Mol C, Mosci S, Traskine M, Verri A: A regularized method for selecting nested groups of relevant genes from microarray data. $J$ Comput Biol 2009, 16:1-15

29. Barla A, Mosci S, Rosasco L, Verri A: A method for robust variable selection with significance assessment. Proceedings of ESANN 2008.

30. Fardin $\mathrm{P}$, Barla A, Mosci S, Rosasco L, Verri A, et al: The I1-I2 regularization framework unmasks the hypoxia signature hidden in the transcriptome of a set of heterogeneous neuroblastoma cell lines. BMC Genomics 2009, 10:474.

31. Jurman G, Merler S, Barla A, Paoli S, Galea A, et al: Algebraic stability indicators for ranked lists in molecular profiling. Bioinformatics 2008 , 24:258-264.

32. Huang DW, Sherman BT, Lempicki RA: Systematic and integrative analysis of large gene lists using david bioinformatics resources. Nat Protoc 2009, 4:44-57.

33. Ashburner M, Ball CA, Blake JA, Botstein D, Butler H, et al: Gene ontology: tool for the unification of biology. the gene ontology consortium. Nat Genet 2000, 25:25-9.

34. Hosack DA, Dennis G, Sherman BT, Lane HC, Lempicki RA: Identifying biological themes within lists of genes with ease. Genome Biol 2003, 4:R70.
35. Salomonis N, Hanspers K, Zambon AC, Vranizan K, Lawlor SC, et al: Genmapp 2: new features and resources for pathway analysis. BMC Bioinforma 2007, 8:217.

36. Kanehisa M, Goto S: Kegg: kyoto encyclopedia of genes and genomes. Nucleic Acids Res 2000, 28:27-30.

37. BioCarta web site, the interactive web-based resources for life scientists. www. biocarta.com.

38. Paquette J, Tokuyasu T: Egan: exploratory gene association networks Bioinformatics 2010, 26:285-6

39. Raso A, Mascelli S, Nozza P, Ugolotti E, Vanni I, et al: Troubleshooting fine-tuning procedures for qpcr system design. J Clin Lab Anal 2011, 25:389-94.

40. Raso A, Negri F, Gregorio A, Nozza P, Mascelli S, et al: Successful isolation and long-term establishment of a cell line with stem cell-like features from an anaplastic medulloblastoma. Neuropathol Appl Neurobiol 2008, 34:306-15.

41. Pfaffl MW: A new mathematical model for relative quantification in real-time rt-pcr. Nucleic Acids Res 2001, 29:e45.

42. Bustin SA: Absolute quantification of mRNA using real-time reverse transcription polymerase chain reaction assays. J Mol Endocrinol 2000, 25:169-93.

43. Fiermonte G, Palmieri L, Todisco S, Agrimi G, Palmieri F, et al: Identification of the mitochondrial glutamate transporter. bacterial expression, reconstitution, functional characterization, and tissue distribution of two human isoforms. J Biol Chem 2002, 277:19289-94.

44. Bustin SA, Benes V, Garson JA, Hellemans J, Huggett J, et al: The miqe guidelines: minimum information for publication of quantitative real-time pcr experiments. Clin Chem 2009, 55:611-22.

45. Hastie T, Tibshirani R, Friedman JH: The elements of statistical learning: data mining, inference, and prediction. Book 2009:745.

46. Irizarry $\mathrm{R}, \mathrm{Wu} \mathrm{Z}$, Jaffee $\mathrm{H}$ : Comparison of affymetrix genechip expression measures. Bioinformatics 2006, 22:789-94.

47. Stieber WW: Low-grade gliomas. Curr Treat Options Oncol 2001, 2(6):495-506.

48. Tatevossian RG, Lawson ARJ, Forshew T, Hindley GFL, Ellison DW, et al: Mapk pathway activation and the origins of pediatric low-grade astrocytomas. J Cell Physiol 2010, 222:509-14.

49. Martynoga B, Morrison H, Price DJ, Mason JO: Foxg1 is required for specification of ventral telencephalon and region-specific regulation of dorsal telencephalic precursor proliferation and apoptosis. Dev Biol 2005 283:113-27.

50. Muzio L, Mallamaci A: Foxg1 confines cajal-retzius neuronogenesis and hippocampal morphogenesis to the dorsomedial pallium. J Neurosci 2005, 25:4435-41.

51. Hanashima C, Li SC, Shen L, Lai E, Fishell G: Foxg1 suppresses early cortical cell fate. Science 2004, 303:56-9.

52. Massagué J: Tgfbeta in cancer. Cell 2008, 134:215-30.

53. Yin XL, Hui ABY, Pang JCS, Poon WS, Ng HK: Genome-wide survey for chromosomal imbalances in ganglioglioma using comparative genomic hybridization. Cancer Genet Cytogenet 2002, 134:71-6.

54. Fukushima T, Katayama Y, Watanabe T, Yoshino A, Komine C, et al: Aberrant tp53 protein accumulation in the neuronal component of ganglioglioma. J Neurooncol 2005, 72:103-6.

55. Schindler G, Capper D, Meyer J, Janzarik W, Omran H, et al: Analysis of braf v600e mutation in 1,320 nervous system tumors reveals high mutation frequencies in pleomorphic xanthoastrocytoma, ganglioglioma and extra-cerebellar pilocytic astrocytoma. Acta Neuropathol 2011, 121:397-405.

doi:10.1186/1471-2407-13-387

Cite this article as: Mascelli et al.: Molecular fingerprinting reflects different histotypes and brain region in low grade gliomas. BMC Cancer 2013 13:387 